\title{
Front Matter: Volume 10457
}

, "Front Matter: Volume 10457," Proc. SPIE 10457, AOPC 2017: Laser Components, Systems, and Applications, 1045701 (21 December 2017); doi: $10.1117 / 12.2300607$

SPIE. Event: Applied Optics and Photonics China (AOPC2017), 2017, Beijing, China 


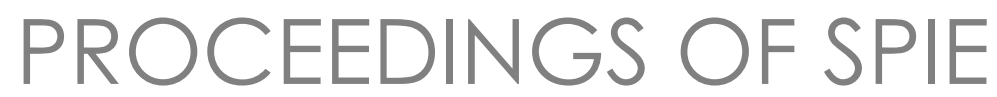

\section{AOPC 2017: Laser Components, Systems, and Applications}

\section{Shibin Jiang \\ Lijun Wang \\ Lan Jiang \\ Long Zhang \\ Editors}

4-6 June 2017

Beijing, China

Sponsored by

SPIE

Chinese Society for Optical Engineering (China)

Organized by

Chinese Society for Optical Engineering (China)

Photoelectronic Technology Committee, Chinese Society of Astronautics (China)

Department of Cooperation and Coordination for Industry, Academe and Research, CHIA (China)

Science and Technology on Low-light-level Night Vision Laboratory (China)

Science and Technology on Electro-Optical Information Security Control Laboratory (China)

Published by

SPIE

Volume 10457

Part One of Two Parts 
The papers in this volume were part of the technical conference cited on the cover and title page. Papers were selected and subject to review by the editors and conference program committee. Some conference presentations may not be available for publication. Additional papers and presentation recordings may be available online in the SPIE Digital Library at SPIEDigitalLibrary.org.

The papers reflect the work and thoughts of the authors and are published herein as submitted. The publisher is not responsible for the validity of the information or for any outcomes resulting from reliance thereon.

Please use the following format to cite material from these proceedings:

Author(s), "Title of Paper," in AOPC 2017: Laser Components, Systems, and Applications, edited by Shibin Jiang, Lijun Wang, Lan Jiang, Long Zhang, Proceedings of SPIE Vol. 10457 (SPIE, Bellingham, WA, 2017) Seven-digit Article CID Number.

ISSN: 0277-786X

ISSN: 1996-756X (electronic)

ISBN: 9781510613959

ISBN: 9781510613966 (electronic)

Published by

SPIE

P.O. Box 10, Bellingham, Washington 98227-0010 USA

Telephone +1 3606763290 (Pacific Time) · Fax +1 3606471445

SPIE.org

Copyright @ 2017 , Society of Photo-Optical Instrumentation Engineers.

Copying of material in this book for internal or personal use, or for the internal or personal use of specific clients, beyond the fair use provisions granted by the U.S. Copyright Law is authorized by SPIE subject to payment of copying fees. The Transactional Reporting Service base fee for this volume is $\$ 18.00$ per article (or portion thereof), which should be paid directly to the Copyright Clearance Center (CCC), 222 Rosewood Drive, Danvers, MA 01923. Payment may also be made electronically through CCC Online at copyright.com. Other copying for republication, resale, advertising or promotion, or any form of systematic or multiple reproduction of any material in this book is prohibited except with permission in writing from the publisher. The CCC fee code is 0277 $786 \times / 17 / \$ 18.00$.

Printed in the United States of America.

Publication of record for individual papers is online in the SPIE Digital Library.

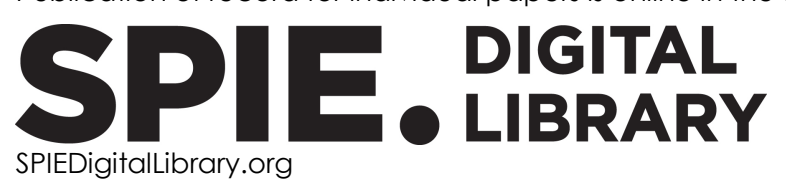

Paper Numbering: Proceedings of SPIE follow an e-First publication model. A unique citation identifier (CID) number is assigned to each article at the time of publication. Utilization of CIDs allows articles to be fully citable as soon as they are published online, and connects the same identifier to all online and print versions of the publication. SPIE uses a seven-digit CID article numbering system structured as follows:

- The first five digits correspond to the SPIE volume number.

- The last two digits indicate publication order within the volume using a Base 36 numbering system employing both numerals and letters. These two-number sets start with 00, 01, 02, 03, 04, 05, 06, 07, 08, 09, OA, OB ... 0Z, followed by 10-1Z, 20-2Z, etc. The CID Number appears on each page of the manuscript. 


\title{
Contents
}

\author{
xi Authors \\ xvii Conference Committee \\ xix Introduction
}

\section{Part One}

\section{LASER COMPONENTS, SYSTEMS, AND APPLICATIONS}

1045702 The effects of pulsed laser parameters on the photoacoustic detection of glucose aqueous solution [10457-3]

1045703 Obstacle detection and avoiding of quadcopter [10457-5]

1045704 Simulation analysis of debris detection and removal by space-based laser [10457-6]

1045705 A large size vertical cavity surface emitting laser with multiple concentric ring apertures [10457-7]

1045706 Advances in high power linearly polarized fiber laser and its application (Invited Paper) [10457-8]

1045707 Optimizing the phase matching in high-order harmonics generation [10457-10]

1045708 Numerical study of temperature in a direct-liquid-cooled Nd:YLF thin disk laser [10457-1 1]

1045709 Twist phase-induced characteristics changes of a radially polarized Gaussian SchellModel beam in a uniaxial crystal orthogonal to the optical axis [10457-12]

10457 OA Raman laser amplifier in methane-filled hollow-core fiber [10457-13]

10457 OB A calibration method of capacitive displacement sensor based on laser interference [10457-15]

10457 OC Complex soliton bunching patterns induced by nonsaturable absorption [10457-16]

10457 OD Spectroscopic properties of nanosecond laser-ablated ZnO: Mg thin film plasma [10457-18]

10457 OE Correction of phase delay caused by mixer and lowpass filter in heterodyne interferometer [10457-19]

10457 OF High-energy master oscillator power amplifier with near-diffraction-limited output based on ytterbium-doped PCF fiber [10457-20] 
10457 OG Efficient self-seeding in a long cavity-length discharge-excited excimer laser system [10457-21]

$10457 \mathrm{OH} \quad$ Experimental investigation of thermal characteristics of erbium doped distributed feedback fiber laser output power [10457-22]

10457 Ol The method for scanning reshaping the spectrum of chirped laser pulse based on the quadratic electro-optic effects [10457-38]

10457 0J Research on an algorithm for visibility based on lidar [10457-39]

10457 OK Test and analysis of background sunlight's influence on laser receiver [10457-42]

$10457 \mathrm{OL} \quad$ Uncertainty analysis of distortion measurement of laser differential confocal by different evaluation methods [10457-43]

10457 OM Dual-wavelength external cavity laser device for fluorescence suppression in Raman spectroscopy [10457-45]

10457 ON Detection on vehicle vibration induced by the engine shaking based on the laser triangulation [10457-46]

$1045700 \quad$ Ultrafast broadband reverse saturable absorption based on two-photon induced singlet state [10457-47]

10457 OP Research on microstructure properties of the TiC/Ni-Fe-Al coating prepared by laser cladding technology [10457-48]

$104570 Q \quad$ Generation of picosecond optical pulse based on chirp compensation [10457-49]

10457 OR Experiment of optical axis angle of electro-optic crystal by conoscopic interference and $x$ ray diffraction method [10457-50]

10457 OS A fiber-laser-pumped four-wavelength continuous-wave mid-infrared optical parametric oscillator [10457-51]

10457 OT Observation of the dispersion of wedge waves propagating along cylinder wedge with different truncations by laser ultrasound technique [10457-52]

10457 OU Time-domain properties of femtosecond laser diffracted by transmitting blazed gratings [10457-53]

10457 OV Investigations on the carbon contaminations on the alkali cells of DPAL with hydrocarbon buffer gas [10457-59]

10457 OW Efficient yellow-light generation based on a Q-switched frequency-doubled self-Raman laser [10457-62]

10457 OX Design the laser wrecker system based on unmanned aerial vehicle [10457-63]

10457 OY Error analysis and experimental verification of a fiber based displacement interferometer [10457-66] 
$10457 \mathrm{OZ}$ Multiwavelength mode-locked cylindrical vector beam fiber laser based on mode selective coupler [10457-67]

1045710 Measurements of loss and gain of optically pumped InGaAs semiconductor lasers based on the photoluminescence spectra from dual facets [10457-68]

1045711 Control algorithms and applications of the wavefront sensorless adaptive optics [10457-70]

1045712 Broadband spatial optical filtering with a volume Bragg grating and a blazed grating pair [10457-82]

1045713 Effects of gap width on droplet transfer behavior in ultra-narrow gap laser welding of high strength aluminum alloys [10457-85]

1045714 Spectrum properties and Judd-Ofelt analysis of $\mathrm{Er}^{3+}$ doped $\mathrm{P}_{2} \mathrm{O}_{5}$-based glasses [10457-86]

1045715 Design and fabrication of lithium niobate based single mode Y-branch APE waveguide [10457-87]

1045716 Development of a fluorescence lidar for biological aerosol detection in the air [10457-89]

1045717 HAZ characteristics of laser cladding remanufacturing ductile iron component [10457-90]

1045718 Fabricating micro-nano structures on stainless steel surface by picosecond laser [10457-92]

1045719 Research on improving the precision of $1064 \mathrm{~nm}$ fiber Doppler lidar in detecting rotating targets [10457-94]

10457 1A Simulation of NLOS (non-line-of-sight) 3D imaging system [10457-96]

10457 1B Random fiber lasers based on artificially controlled backscattering fibers [10457-98]

10457 1C Single higher-order mode ring-shaped VCSEL with surface relief [10457-102]

10457 ID Analysis of polarization-sensitive resonator based on vectorial eigenvector method [10457-103]

10457 1E Preliminary study of the influence of polarization orientation on bulk damage resistances of doubler KDP crystals [10457-104]

10457 IF Study on double end-pumped high power slab laser with laser diode arrays [10457-107]

10457 1G Optical amplification in Er:Yb co-doped fiber and their applications in fiber laser [10457-108]

$104571 \mathrm{H} \quad$ Cylinder surface test with Chebyshev polynomial fitting method [10457-109]

10457 II Fiber up-tapering and down-tapering for low-loss coupling between anti-resonant hollowcore fiber and solid-core fiber [10457-1 11 ] 
$104571 \mathrm{~J}$ A study on the transmission characteristics of laser in the rain by utilizing Modtran software [10457-112]

10457 IK Research on Rb-DPAL for the pumping of SERF rubidium magnetometers [10457-113]

10457 IL Simulation of dissipative-soliton-resonance generation in a passively mode-locked Ybdoped fiber laser [10457-120]

$104571 \mathrm{M} \quad$ Ultrafast pulse erbium-doped fiber laser with a graphene/WS $\mathbf{2}_{\mathbf{2}}$ heterostructure saturable absorber [10457-121]

10457 iN Simulation of laser scattering by typical marine aerosol [10457-123]

1045710 Improved power and efficiency for tapered lasers with optimized photonic crystal structures [10457-125]

10457 IP Applications of OALCLV in the high power laser systems [10457-126]

10457 1Q Hundred-watt level highly stable passively Q-switched fiber laser based on graphene saturable absorber [10457-127]

10457 IR Estimation of mode instability threshold based on local thermal load [10457-128]

10457 is Research on temperature characteristics of laser energy meter absorber irradiated by ms magnitude long pulse laser [10457-129]

\section{Part Two}

10457 IT Generation of high-output yellow light by intracavity doubling Nd:YAG-Nd:YVO 4 hybrid gain Raman laser [10457-131]

10457 iU Study on modulation amplitude stabilization method for PEM based on FPGA in atomic magnetometer [10457-132]

$104571 \mathrm{~V}$ Comparison of SP-LIBS and DP-LIBS on metal and non-metal testing based on LIBS [10457-134]

10457 IW Measurement of the wavefront distribution characteristics of a 400-1700nm supercontinuum light source [10457-135]

10457 1X Heat-affected zone microstructure and mechanical properties evolution for laser remanufacturing LZ50 axle steel [10457-136]

10457 IY Efficient phase locking of two dual-wavelength fiber amplifiers by an all-optical selffeedback loop [10457-137]

1045712 An expander system of high stable laser beam for cesium atomic fountain clocks [10457-138]

1045720 Interaction of ultrashort pulse with two-level medium beyond the slowly varying envelope approximation [10457-142] 
1045721 Theoretical study on the absorption features of a diode side-pumped alkali laser [10457-143]

1045722 The ultra-stable microwave based on ultra-stable laser [10457-145]

1045723 Laser beam shaping design based on micromirror array [10457-146]

1045724 Subsurface damage distribution and processing method of ground fused silica [10457-147]

1045725 Diffraction characteristics of hump volume Bragg grating in photo-thermo-refractive glass [10457-148]

1045726 Output characteristics of the mode-locked thulium-doped fiber laser near $2 \mu \mathrm{m}$ based on

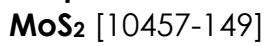

1045727 Studies on low-loss coupling of non-node anti-resonant hollow-core fiber and tapered fiber [10457-150]

1045728 Intensity and frequency stabilization of a laser diode by simultaneously controlling its temperature and current [10457-153]

1045729 Dependence of output features of a micro-cavity laser on the cavity structure [10457-154]

10457 2A Influence of axial temperature distribution to optical parametric gain in CW-OPO [10457-155]

10457 2B Effect of ridge structure on electro-optical characteristics of ridge-waveguide lasers with low vertical divergence based on photonic crystal structure [10457-156]

$104572 \mathrm{C}$ Study on on-machine defects measuring system on high power laser optical elements [10457-157]

10457 2D An accurate method for investigation of laser-induced damage of optical component at $351 \mathrm{~nm}$ [10457-158]

$104572 \mathrm{E} \quad \mathbf{2} \boldsymbol{\mu m}$ all fiber multi-wavelength $\mathrm{Tm} / \mathrm{Ho}$ co-doped fiber laser [10457-160]

10457 2F Multi-wavelength Tm/Ho co-doped mode-locked fiber laser with a simple cavity [10457-161]

$104572 \mathrm{G}$ The coherent combination of multi-wavelength fiber laser [10457-162]

$104572 \mathrm{H}$ Investigation of numerical simulation on all-optical flip-flop stability maps of $1550 \mathrm{~nm}$ vertical-cavity surface-emitting laser [10457-163]

$1045721 \quad$ Mode-locked $2 \mu \mathrm{m}$ fiber laser with a Tm/Ho-doped fiber saturable absorber [10457-164]

10457 2J High-efficiency fiber-coupled module based on multi-single emitter photonic-band-crystal laser diodes [10457-165]

10457 2K Multimode fiber modal decomposition based on hybrid genetic global optimization algorithm [10457-166] 
10457 2L Passively q-switched fiber lasers based on concave gold bipyramids saturable absorbers [10457-167]

$104572 \mathrm{M}$ Integrated double-clad photonic crystal fiber amplifier [10457-168]

$104572 \mathrm{~N} 250 \mathrm{~W}$ continuous-tunable all-fiberized single-frequency polarization-maintained amplifiers with wavelength spanning from $1065 \mathrm{~nm}$ to $1090 \mathrm{~nm}$ [10457-169]

1045720 Study on VCSEL laser heating chip in nuclear magnetic resonance gyroscope [10457-170]

10457 2P Analysis of detection performance of multi band laser beam analyzer [10457-172]

$104572 Q$ Suppression of high order modes employing active self-imaging mode filter in large mode area strongly pumped fiber amplifier [10457-174]

$104572 R \quad$ Ultra-long-period grating as a novel tool for multi-wavelength ultrafast photonics [10457-176]

1045725 A homogeneous cooling scheme investigation for high power slab laser [10457-177]

10457 2T A single-longitudinal-mode Tm, Ho:YAG laser [10457-178]

$104572 \mathrm{U}$ Laser frequency-offset locking based on the frequency modulation spectroscopy with higher harmonic detection [10457-179]

$104572 \mathrm{~V}$ Compensation for the phase-type spatial periodic modulation of the near-field beam at $1053 \mathrm{~nm}$ [10457-180]

10457 2W Control of metal ablation by temporally shaped femtosecond laser pulse [10457-181]

10457 2X Characteristic analysis of atmospheric boundary layer and particulate matter in Beijing [10457-182]

10457 2Y PT-symmetry of coupled fiber lasers [10457-184]

$104572 Z$ Nanosecond pulse pumped, fiber-integrated narrow linewidth linearly polarized Raman amplifier at $1120 \mathrm{~nm}$ [10457-185]

1045730 Design of laser diode driver with constant current and temperature control system [10457-186]

1045731 Research on method of obtaining multi wavelength correction coefficient of laser power meter [10457-187]

1045732 The entangled photons generation in third order nonlinearity of spontaneous parametric down conversion by whispering gallery mode resonator [10457-189]

1045733 Localization in disordered potential in photonic lattice realized in time domain [10457-191]

1045734 Photonic modes in synthetic photonic lattices localized due to nontrivial gauge field circulation [10457-192] 
1045735 Verification and calibration of laser Doppler flowmetry (LDF) prototype for measurement of microcirculation [10457-193]

1045736 Monolithic wavelength-tunable thulium-doped fiber laser mode-locked by semiconductor saturable absorber mirror with 120nm tuning range [10457-194]

1045737 Ultra-long duration and ultra-high duty cycle dissipative soliton resonance in a modelocked thulium-doped fiber laser [10457-195]

1045738 Effects of $\mathrm{Y}^{3+}$ codoping on the spectral properties of Nd:CaF2 crystals [10457-197]

1045739 Effects of $\mathbf{G d}^{3+}$ on the photoluminescence properties of $\mathrm{Nd}^{3+}$-doped $\mathrm{SrF}_{2}$ crystal [10457-198]

10457 3A Modulated visible spectra properties of $\operatorname{Pr} \mathrm{Ca}_{1-\times} \mathbf{R}_{\mathbf{x}} \mathrm{F}_{2+\times}(\mathrm{R}=\mathrm{Y}, \mathrm{La}, \mathrm{Gd})$ crystals [10457-199]

10457 3B Modeling the mode competition of $\mathrm{Yb}$-doped fiber amplifiers in consideration of photodarkening [10457-200]

10457 3C Compact and efficient blue laser sheet for measurement [10457-202]

10457 3D Analysis of thermal effects in high power Yb doped fiber amplifier by distributed pumping [10457-204]

10457 3E Theoretical study of Raman fiber laser and random fiber laser [10457-205]

10457 3F Simultaneous three wavelength mid-IR laser by utilizing MgO:PPLN crystal [10457-206]

$104573 G$ Coaxial monitoring of temperature field in selective pulsed laser melting [10457-207] 
Proc. of SPIE Vol. 10457 1045701-10 Downloaded From: https://www.spiedigitallibrary.org/conference-proceedings-of-spie on 26 Apr 2023
Terms of Use: https://www.spiedigitallibrary.org/terms-of-use 


\section{Authors}

Numbers in the index correspond to the last two digits of the seven-digit citation identifier (CID) article numbering system used in Proceedings of SPIE. The first five digits reflect the volume number. Base 36 numbering is employed for the last two digits and indicates the order of articles within the volume. Numbers start with 00, 01, 02, 03, 04, 05, 06, 07, 08, 09, OA, OB...0Z, followed by 10-12, 20-2Z, etc.

\author{
An, Guofei, 21, 29 \\ An, Honghai, $1 \mathrm{P}$ \\ Ao, Guanghong, 00 \\ Ba, Rongsheng, 1E, 2D \\ Bai, Gang, 2Q \\ Bai, Xiaolei, IG \\ Bai, Yong-lin, 07 \\ Bhattacharjee, Nilabha, 07 \\ Bian, Jintian, OV \\ Cai, Chao, 24 \\ Cai, Chenguang, $\mathrm{OE}$ \\ Cai, He, 21, 29 \\ Cai, Xiaotian, 2A \\ Cai, Yu, $0 Z$ \\ Cai, Zhijian, OM \\ Cao, Hongzhong, $3 G$ \\ Cao, Jingming, 32 \\ Cao, Pengfei, 09 \\ Cao, Shiying, 22 \\ Chai, Liqun, 1E \\ Chang, Hao, 04 \\ Chen, Bo, 1E, 2D \\ Chen, Bo, 39 \\ Chen, Daru, 1B \\ Chen, Fang, 2T \\ Chen, Guanjin, 12 \\ Chen, Huai-xi, 15 \\ Chen, Jiang, 12 \\ Chen, Jinbao, 2K, 3B \\ Chen, Keshan, 1Y, 2G \\ Chen, Qianrong, OK \\ Chen, Weicheng, OC \\ Chen, Wenxue, ON \\ Chen, $\mathrm{Xi}, 1 \mathrm{H}$ \\ Chen, Xiaolong, $2 Q$ \\ Chen, Xiaomei, 2P \\ Chen, Yang, 2J \\ Chen, Yanzhong, 2S \\ Chen, Yubin, OA \\ Chen, Zhongyun, 3G \\ Chen, Zilun, 2M \\ Cheng, $\mathrm{He}, \mathrm{IP}$ \\ Cheng, Yu, IP \\ Cheng, Zhen, OD \\ Choge, Dismas K., 15 \\ Churkin, Dmitry V., 2Y, 33, 34 \\ Cui, Huirong, OG \\ Cui, Lei, 31 \\ Dai, Shaoyang, 22
}

Ding, Lei, 1E, 2D

Ding, Xin, OW, $1 T$

Dong, Shiyun, 13, 17, 18, 1X

Dong, Xinzheng, 26

Dong, Yanbing, $0 \mathrm{~J}$

Dou, Zhiyuan, 36, 37

Du, Baolin, 2P

Du, Shaojun, IW

Du, Wenxiong, 1L, 1M

Duan, Lihong, $1 \mathrm{U}$

Fan, Chengyu, is

Fan, Lianwen, $2 S$

Fan, Wei, OF, IP

Fan, Xiaoli, 23

Fan, Yuanyuan, OG

Fan, Zhen-fang, 30

Fan, Zhongwei, $2 S$

Fang, Fang, 22

Fang, Han, 23

Fang, Jinxiang, $1 X$

Feng, Yuan, 05, $1 \mathrm{C}$

Feng, Xiangyi, $1 \mathrm{X}$

Feng, Yanying, $2 U$

Fu, Shijie, 3D, 3E

Fu, Wenyu, 09

Gang, Wang, 24

Gao, Changsheng, OX

Gao, Fan, OU, 25

Gao, Yaru, 2V

Ge, Wenqi, $2 S$

Gu, Xiaohui, 21

Gu, Yanran, 2M

Guo, Bo, 2R

Guo, Hao, OK

Guo, Jian, $\mathrm{OH}$

Guo, Linyang, 39

Guo, Pei-ji, $1 \mathrm{H}$

Han, Chongchong, OU

Han, Gaoce, OV

Han, Juhong, 21, 29

Han, Qingbang, OT

Han, Tiegiang, $\mathrm{ON}$

Han, Yanbing, $0 \mathrm{O}$

Hao, Yong-Qin, 05, 1C

$\mathrm{He}$, Bing, $2 \mathrm{Q}$

$\mathrm{He}$, Jianguo, $2 \mathrm{~S}$

He, Jiawu, 13, 17, 18

He, Junfang, OD

He, Peng, 13, 17 
He, Tingyao, 16

He, Xiang, 24

Hou, Jing, 36, 37

$\mathrm{Hu}$, Chia-Ming, 35

Hu, Haojun, $1 Y$

Hu, Leili, 2P

Hu, Mengting, 26

$\mathrm{Hu}$, Qing, 24

$\mathrm{Hu}$, Xinhan, $\mathrm{ON}$

Hu, Yaocheng, ON

Hu, Youyou, ID

Hu, Zhangfang, 2

Hu, Zhaohui, 28

Huang, Anping, 14

Huang, Dajie, 1P

Huang, Liangjin, 3B

Huang, Long, 06

Huang, Ping, $\mathrm{OZ}$

Huang, Wei, OV, IK

Huang, Zhen, 02

Huo, Yi-wei, $1 \mathrm{~N}$

Jia, Dan, $2 S$

Jia, Jing, OT

Jia, Y., 10

Jia, Ya-qing, 31

Jia, Yuchen, 20

Jiang, Dapeng, 38, 39

Jiang, Hongzhen, OR

Jiang, Pengbo, OW, $1 \mathrm{~T}$

Jiang, Qiuxia, 2E, 2F

Jiao, Junke, $O P$

Jin, Xing, 04, OB

Jing, Wang, 23

Jing, Xueping, OT

Ju, Youlun, 2T

Kang, Bo-kun, 04

Kang, Yi-fan, 07

Ke, Weiwei, $1 \mathrm{R}$

Kim, Dong Eon, 07

Lan, Changyong, IM

Lei, Bing, $1 Y$

Lei, Jian, $1 \mathrm{~F}$

Leng, Jinyong, 1Q, 2K, 3B

Li, Ai-jun, $1 \mathrm{~J}$

Li, Bao, $1 \mathrm{~F}$

Li, Bo, OK

Li, Bo, ID

Li, Chonghe, 18

Li, Chun, $1 \mathrm{M}$

Li, Dong, OR

Li, Dun, IW

Li, Guang-wei, 15

Li, Heping, 1L, 1M

Li, Hui, OG

Li, Hui, OV

Li, Jie, 1E, 2D

$\mathrm{Li}$, Jun, $2 \mathrm{H}$

Li, Lei, 2K, 3B

Li, Li, 2T

Li, Miao, 3B
Li, Nan, $1 \mathrm{~S}$

Li, Nanlei, OB

Li, Rao, OF

Li, Shangyuan, $0 Q$

Li, Shuli, OD

Li, Tenglong, IR

Li, Tianchu, 22

Li, Tianqi, $1 \mathrm{~F}$

Li, Wenxiu, 14

Li, Xia, OJ

Li, Xian-tao, $1 \mathrm{~N}$

Li, Xiao, OS, 2A

Li, Xin, $1 G$

Li, Xuechun, 1P

Li, YaJun, 1E

Li, Yang, 05

Li, Yongjian, 17

Li, Yung-Hui, 35

Li, Zhaoning, OY

Li, Zhihong, 36, 37

Li, Zhiyong, OV, $1 \mathrm{~K}$

Liang, Wan-guo, 15

Liang, Weiwei, OK

Liang, Xiaoyang, 20

Lin, Jiajian, 03

Lin, Jingjun, IV

Lin, Rui, 12

Lin, Weiran, 2S

Lin, Xiaomei, $1 \mathrm{~V}$

Lin, Yuzhe, 10, 2B

Lin, Zhifan, 2C

Lin, Zunqi, OF, $1 \mathrm{P}$

Liu, Che, 3G

Liv, Chong, 08

Liv, Cong, 1L, 1M

Liu, Dandan, $1 Z$

Liu, Dean, 2V

Liv, Guodong, 02

Liu, Guo-Jun, 05

Liv, Hao, OJ

Liv, Hao, $2 S$

Liu, Jiaguo, 23

Liu, Jiaming, 14

Liu, Jian, OW, $1 T$

Liu, Jun, $2 M$

Liu, Nianfeng, 22

Liu, Ningwen, OY

Liu, Qinyong, 1R

Liu, Wenbo, 27

Liu, Xingrun, OJ

Liu, $X \mathrm{U}, \mathrm{OR}$

Liu, Yakun, 2N

Liu, Yang, $1 \mathrm{~F}$

Liu, Yong, OR

Liv, Yong, 1L, 1M

Liu, Yue-hao, 19

Liu, Zhihua, OE

Lu, Long-zhao, 20

LU, Qisheng, 0A

LU, W., 10 
Luo, Aiping, OC

Luo, Chi, 2C

Ma, Fengkai, 38, 39, 3A

$\mathrm{Ma}, \mathrm{H} \cup \mathrm{a}, \mathrm{OL}$

Ma, Liang, 11

$\mathrm{Ma}, \mathrm{Na}, \mathrm{OK}$

Ma, Pengfei, 06, 2N

Ma, Ping, 24

Ma, Xiao-Hui, 05, $1 \mathrm{C}$

Ma, Xiaolong, 10, 2J

$\mathrm{Ma}, \mathrm{Yi}, \mathrm{IR}$

Makarenko, Maxim, $2 Y$

Mei, Bingchu, 39

Meng, Zhixin, $2 \mathrm{U}$

Mo, Zeqiang, $2 S$

Mu, Weiwei, 28

$\mathrm{Na}$, Jin, $1 \mathrm{E}$

$\mathrm{Ni}$, Zhibin, ON

Ning, Y.-Q., 10

Pan, Fusheng, $1 X$

Pang, Siyuan, 38

Pankov, Artem, 34

Peng, Gang-Ding, $\mathrm{OH}$

Peng, Wanjing, IR

Qi, Aiyi, 2B

Qi, Hai-feng, $\mathrm{OH}$

Qi, Yan, 3C, 3F

Qi, Yunfeng, 2Q

Qian, Xiaobo, 38, 39, 3A

Qiang, Zexuan, 1B

Qiao, Chunhong, 1S

Qiao, Zhi, OF

Qu, Hongwei, 1O, 2B, 2J

Quan, Wei, $1 \mathrm{U}$

Rao, Zhimin, 16

Ren, Huan, $\mathrm{OL}$

Ren, Zhong, 02

Rong, Kepeng, 21, 29

Ruan, Jun, $1 Z$

Shang, Yaping, OS

Shang, Zhen, $2 X$

She, Lijuan, 1B

Shen, Jianfei, 26

Shen, Shengnan, 1L, 1M

Sheng, Liyuan, OP

Sheng, Quan, OW, 1T, 3D, 3E

Shi, Feng, 2C

Shi, Guang, 00

Shi, Jianhua, IY

Shi, Junru, 1 Z

Shi, Rui, OW

Shi, Wei, 1G, 3D, 3E

Shi, Zhendong, OL

Si, Lei, $2 \mathrm{~N}$

Smirnov, Sergey $V_{\text {., }} 2 Y$

Song, Chaoqun, 13

Song, Haizhi, 29

Song, Jiaxin, 1Q, 2L

Song, Xingliang, $0 G$

Song, Yanrong, 26
Song, Yinglin, 00

Song, Zhi-qiang, $\mathrm{OH}$

Su, Bida, 23

Su, Liangbi, 38, 39, 3A

Su, Rongtao, 06, 2N, $2 Z$

Su, Xiuqin, $1 \mathrm{~A}$

Suchkov, Sergey V., $2 Y$

Sukhorukov, Andrey A., 2Y, 33, 34

Sun, Bing, OW

Sun, Haoran, IV

Sun, Hong-hui, $1 \mathrm{~J}$

Sun, Xiang-lin, 31

Sun, Xiaofeng, $O Q$

Sun, Xiaojie, 12

Sun, Yinhong, 1R

Tan, Hang, OI

Tan, Jingjing, $1 \mathrm{~A}$

Tan, Min, $2 \mathrm{X}$

Tan, Rongqing, OV, $1 \mathrm{~K}$

Tang, Chun, $1 R$

Tang, Ruyu, 2V

Tang, Xiahui, 1D

Tang, Xiaojun, $1 \mathrm{~F}$

Tao, Shixing, OY

Tian, Bao-lu, 15

Tian, Chengcai, 14

Tian, Jinrong, 26

Tikan, Alexey M., 33

Tsai, Ming-Lun, 35

Vatnik, llya D., 2Y, 33, 34

Wang, Anqi, $2 U$

Wang, Bangxin, $2 X$

Wang, Biao, $1 \mathrm{~N}$

Wang, Bin, 11

Wang, Bin, 18

Wang, Chang, $\mathrm{OH}$

Wang, Chao, 07

Wang, Chao, IP

Wang, Dapeng, OB

Wang, Dizhong, 03

Wang, Guilin, 2C

Wang, Hongying, OD

Wang, Hongyuan, 21, 29

Wang, Jiangfeng, IP

Wang, Jing, 28, 20

Wang, Jingya, 39, 3A

Wang, Ke, 08

Wang, Ming-cai, 30

Wang, Peng, OS

Wang, Qinghua, $1 \mathrm{U}$

Wang, Shunyan, 21, 29

Wang, Wei-tao, $\mathrm{OH}$

Wang, Xia, 1C

Wang, Xiaochao, OF

Wang, Xiaofa, 2E, 2F, 2H, 2

Wang, Xiaoliang, 1B

Wang, Xiaolin, 06, 2N, $2 Z$

Wang, Xin, 25

Wang, Yanshan, $1 R$

Wang, Yanwei, 3C, 3F 
Wang, Ying, OE

Wang, Yingjian, $2 X$

Wang, Yi-shan, 07

Wang, You, 21, 29

Wang, $Y u, 3 C$

Wang, Yufei, 2B, 2J

Wang, Yujie, OV, IK

Wang, Yunfeng, ON

Wang, Yuxiao, 00

Wang, Zefeng, 0A, 11, 27

Wang, Zhi-guo, 30

Wang, Zhi-Wei, 05, 1C

Wei, Hui, IP

Wei, Zhaoqi, $1 \mathrm{~A}$

Wen, Da-qing, 20

Wu, Bin, 3C

Wu, Hanshuo, 1Q, 2L

Wu, J., 10

WU, Jian, 1Q, 2L

WU, Jianhong, OM

Wu, Jingyao, $1 \mathrm{~A}$

Wu, Qinghui, 3A

Wu, Wenfeng, 20

Wu, Wuming, $1 \mathrm{~W}$

Wu, Xianyun, OI

Wu, Xingzhi, 00

Wu, Yunfeng, OY

$\mathrm{Xi}$, Xiaoming, 1I, 27

Xia, Gang, IP

Xia, Qing, 2H

Xiao, $\mathrm{HU}, 1 \mathrm{Q}$

Xiao, Jinchong, 00

Xiao, Zhisong, 14

Xiaver, Jolly, 32

Xie, ChenBo, $2 X$

Xie, Jianlai, $1 \mathrm{C}$

Xie, Zhaoxin, 3D, 3E

Xie, Zhiwei, 18

$X U$, Binshi, 13, 17, 1X

$X U$, Honglei, 1E, 2D

$X U$, Jiangming, 06, 1Q, $2 L$

$X u$, Jia-ting, $1 \mathrm{~J}$

$X \cup$, Jiayue, 38

$X \cup$, Jun, $1 \mathrm{~J}$

$X U$, Jun, 3A

Xu, Lu, 19

$X \cup$, Qingshan, OJ

$X u$, Runqin, 26

$X \cup$, Xianfan, 14

Xu, Xiaojun, OS, 36, 37

$X \cup, Y i-b i n, 15$

$X \cup, Z h \cup o, 36,37$

$X \cup, Z i f a, O P$

Xue, Xia, 14

Xue, Xiaoxiao, $O Q$

Yan, Boxia, 3C, 3F

Yan, Chang-Ling, 05

Yan, Shixing, 13, 17, 18, 1X

Yang, Aihua, 2V

Yang, Biwu, ON
Yang, Cheng-hua, 19

Yang, Gaochao, is

Yang, Huizhen, 11

Yang, Jiaqian, $0 Q$

Yang, Junyi, $0 O$

Yang, Kai-yong, 30

Yang, Lijia, $2 Z$

Yang, Ming, OE

Yang, Wen-Lei, 2R

Yang, XiaoYu, $1 \mathrm{E}$

Yang, $X \cup, 19$

Yang, Xue, $1 \mathrm{~F}$

Yang, $Y i, O L$

Yang, Yifeng, 2Q

Yao, Jianquan, OW, 1T, 3D, 3E

Yao, Tianfu, $1 Y$

Ye, Qing, oV

Ye, Rong, 0 I

Ye, Zhinbin, 08

Yin, Ming, 0 I

Yin, Ruiguang, OK

Yu, Fengxiang, $1 Z$

Yu, Hang, 21, 29

Yu, Hao, 3A

$Y u$, Jin, $2 S$

Yu, Kui-bang, $1 \mathrm{H}$

YU, Q.-N., 10

Yu, Xiang-yang, 20

Yu, Xuanyi, OW, $1 T$

YU, Zhangwei, 1B

Yuan, Jing, 1E, 2D

Yuan, Quan, OL

Yuan, Xiao, OU, 12, 25

Yue, Guang-Li, 05

Zan, Shaoping, OP

Zeng, Lvming, 02

Zha, Congwen, $1 \mathrm{R}$

Zhang, Guiju, 12

Zhang, Guizhong, OW, $1 T$

Zhang, Hanwei, $2 \mathrm{~N}$

Zhang, Hao, 14

Zhang, Hui, $1 \mathrm{Z}$

Zhang, J., 10

Zhang, Jiabo, $2 \mathrm{~W}$

Zhang, Jian-liang, 31

Zhang, Jinghui, is

Zhang, Junhong, 2E

Zhang, Kaihu, 2W

Zhang, Lijia, OG

Zhang, Lin, OL

Zhang, Naiqian, 11, 27

Zhang, Ning, 2W

Zhang, Pengfei, $2 Z$

Zhang, Shangjian, $1 \mathrm{~L}$

Zhang, Shougang, 1 Z

Zhang, Tong, 2C

Zhang, Wei, 21, 29

Zhang, Wenwu, OP

Zhang, Wu, ON

Zhang, X., 10 
Zhang, Xiang, OU, 25

Zhang, Xin, 05

Zhang, Xin, OX

Zhang, Xueru, 00

Zhang, Xuting, OM

Zhang, Yong, 19

Zhang, YU, OT

Zhang, Yufang, 2J

Zhang, Zuxing, $\mathrm{OZ}$

Zhao, Chenghai, IW

Zhao, Guomin, 2A

Zhao, Heng, 24

Zhao, Shaoyu, 1O, 2B

Zhao, Xiang, 2Q

Zhao, Xiaoxia, OD

Zhao, Xincai, OY

Zhao, Xuan-ke, $1 \mathrm{~J}$

Zhao, Yuan, 19

Zheng, Fanglan, OR

Zheng, WanGuo, IE

Zheng, Wanhua, 1O, 2B, 2J

Zheng, Xiaoping, $0 Q$

Zheng, Ye, 2Q

Zheng, Yinbo, 1E, 2D

Zhou, Bingkun, $0 Q$

Zhou, Binquan, 28, 20

Zhou, Jianhong, $3 G$

Zhou, Jun, 2Q

Zhou, Pu, 06, 1Q, 2K, 2L, 2Z, 3B

Zhou, Weicai, $O X$

Zhou, Weijing, OB

Zhou, Xinda, 1E, 2D

Zhou, Xuyan, 1O, 2B

Zhou, Yi, OG

Zhou, Yuanshen, 11

Zhu, Haijiang, OE

Zhu, Jianqiang, $2 \mathrm{~V}$

Zou, Yonggang, $1 \mathrm{C}$ 
Proc. of SPIE Vol. 10457 1045701-16 Downloaded From: https://www.spiedigitallibrary.org/conference-proceedings-of-spie on 26 Apr 2023
Terms of Use: https://www.spiedigitallibrary.org/terms-of-use 


\title{
Conference Committee
}

\author{
Conference Chairs
}

Guangjun Zhang, Beihang University (China)

Byoungho Lee, Seoul National University (Korea, Republic of)

\section{Conference Committee}

Desheng Jiang, Wuhan University of Technology (China)

Hequan Wu, Chinese Academy of Engineering (China)

Jianquan Yao, Tianjin University (China)

Jianwei Pan, University of Science and Technology of China (China)

Junhao Chu, Shanghai Institute of Technical Physics, CAS (China)

Junen Yao, Beihang University (China)

Lijun Wang, Changchun Institute of Optics, Fine Mechanics and Physics, CAS (China)

Lin Li, The University of Manchester (United Kingdom)

Liwei Zhou, Beijing Institute of Technology (China)

Min Gu, RMIT University (Australia)

Shibin Jiang, AdValue Photonics Inc. (United States)

Toyohiko Yatagai, Utsunomiya University (Japan)

Wei Wang, Beijing Institute of Aerospace Control Devices, CASC (China)

Weidou Ni, Tsinghua University (China)

Zuyan Xu, Technical Institute of Physics \& Chemistry, CAS (China)

Program Committee

Anand Krishna Asundi, Nanyang Technological University (Singapore)

Bing Zhao, Jilin University (China)

Byoungho Lee, Seoul National University (Korea, Republic of)

Carl Nardell, Terra Bella (United States)

Chunhua Shen, The University of Adelaide (Australia)

Haimei Gong, Shanghai Institute of Technical Physics, CAS (China)

Honghai Liu, University of Portsmouth (United Kingdom)

Huaidong Yang, Tsinghua University (China)

Huijie Zhao, Beihang University (China)

Jannick Rolland, Institute of Optics, University of Rochester (United States)

Jin Lu, Tianjin Jinhang Institute of Technical Physics (China)

Jin Yu, Université Claude Bernard Lyon 1 (France)

Jinxue Wang, SPIE

Lijun Wang, Changchun Institute of Optics, Fine Mechanics and Physics, CAS (China)

Lin Li, The University of Manchester (United Kingdom)

Lan Jiang, Tsinghua University (China) 
Long Zhang, Shanghai Institute of Optics and Fine Mechanics, CAS (China)

Mengxia Xie, Beijing Normal University (China)

Min Gu, RMIT University (Australia)

Min Qiu, Zhejiang University (China)

Shibin Jiang, AdValve Photonics Inc. (United States)

Suijian Xue, National Astronomical Observatories, CAS (China)

Tsutomu Shimura, The University of Tokyo (Japan)

Wei Hang, Xiamen University (China)

Wei Wang, Beijing Institute of Aerospace Control Devices of CASC (China)

Weibiao Chen, Shanghai Institute of Optics and Fine Mechanics, CAS

(China)

Wolfgang Osten, Universität Stuttgart (Germany)

Xiandeng Hou, Sichuan University (China)

Xiangping Li, Jinan University (China)

Xiaocong Yuan, Shenzhen University (China)

Xiaodi Tan, Beijing Institute of Technology (China)

Yadong Jiang, University of Electronic Science and Technology of China (China)

Yanbiao Liao, Tsinghua University (China)

Yong Bi, Academy of Opto-Electronics, CAS (China)

Yongtian Wang, Beijing Institute of Technology (China)

Zhe Wang, Tsinghua University (China)

Zhiping Zhou, Peking University (China)

Session Chairs

1 Mode-Locked Lasers

Lijun Wang, Changchun Institute of Optics, Fine Mechanics and Physics, CAS (China)

Pu Zhou, National University (China)

2 Lasers and Optical Nonlinearity

Zhiyi Wei, Institute of Physics, CAS (China)

Lan Jiang, Beijing Institute of Technology (China)

3 Advanced Materials

Long Zhang, Shanghai Institute of Optics and Fine Mechanics, CAS (China)

Wentao Hu, QPC Lasers (United States) 


\section{Introduction}

Applied Optics and Photonics China (AOPC2017) is the annual conference of the CSOE, and one of the largest academic and industry activities in the field of optical and optoelectronic technology in China. The organization committee has built a platform of academic exchanges, industry exhibitions, and cooperation negotiations in one. There are 8 technical conferences, 7 themes of the Exhibition and approximately 600 technical presentations. We sincerely hope that the research and development of optoelectronic technology are promoted, and the international cooperation between industry and the optical and optoelectronic fields are enhanced.

AOPC2017 is technically co-sponsored by the Chinese Society for Optical Engineering, the Optical Society of Korea (OSK), Optics and Photonics Society of Singapore (OPSS), European Optical Society (EOS), Optical Society of Japan (OSJ) and SPIE. There are also 60 cooperative organizers to support the conference. We received over 1209 contributions from more than 15 countries, including the United States, the United Kingdom, Germany, France, Spain, Australia, Canada, Mexico, Brazil, Japan, Korea, Thailand, Singapore, the Russian Federation, China, and more. There are more than 700 presentations published in the Proceedings of SPIE. After careful discussion, we suggested four keynote speeches which are presented by famous scientists from Germany, Australia, Japan and China. 138 excellent invited talks were presented, 45 are from outside of China. Their presentations reflect first-class research in the field of optics and photonics technology. On behalf of the Organization Committee of AOPC, I express thanks to all the invited speakers and authors for their contributions and support of the conference.

Finally, on behalf of Prof. Zhuang Songlin, and other co-chairmen, and the Organization Committee of AOPC, I would like to heartily thank our sponsors and cooperating organizers for all they have done for the conference, the participants and friends for their interests and efforts in helping us to make the conference a success, the program committee for their effective work and valuable advice, and especially the AOPC2017 Secretariat and the staff of SPIE for their tireless effort and outstanding services in preparing the conference and publishing the Proceedings.

We wish AOPC2017 great success! Hope to see you next year!

\section{Guofan Jin}


Proc. of SPIE Vol. 10457 1045701-20 Downloaded From: https://www.spiedigitallibrary.org/conference-proceedings-of-spie on 26 Apr 2023
Terms of Use: https://www.spiedigitallibrary.org/terms-of-use 